\title{
Et si l'on utilisait HTML... ?
}

Réflexions et premières expériences d'un projet en initiation à la linguistique

\section{Albert Raasch et Björn Kettner}

\section{(2) OpenEdition}

\section{Journals}

Édition électronique

URL : http://journals.openedition.org/ries/2925

DOI : $10.4000 /$ ries. 2925

ISSN : 2261-4265

Éditeur

Centre international d'études pédagogiques

\section{Édition imprimée}

Date de publication : 1 juin 1998

Pagination : 93-100

ISSN : 1254-4590

\section{Référence électronique}

Albert Raasch et Björn Kettner, « Et si l'on utilisait HTML... ? », Revue internationale d'éducation de Sèvres [En ligne], 18 I juin 1998, mis en ligne le 19 avril 2013, consulté le 04 novembre 2019. URL : http:// journals.openedition.org/ries/2925; DOI : 10.4000/ries.2925

Ce document a été généré automatiquement le 4 novembre 2019

(c) Tous droits réservés 


\title{
Et si l'on utilisait HTML...?
}

\author{
Réflexions et premières expériences d'un projet en initiation à la \\ linguistique
}

Albert Raasch et Björn Kettner

\section{La situation de départ}

Les étudiants en "Études romanes ", comme tous les étudiants de philologie en Allemagne, reçoivent une formation linguistique, appelée en général « Initiation à la linguistique ».

Ces cours de base constituent une initiation à la description de la langue (maternelle ou étrangère, d'après les matières choisies) afin que les étudiants qui sont, en général, de futurs professeurs puissent enseigner la langue de leur choix en se fondant sur une compréhension approfondie des mécanismes et structures linguistiques.

Étant donné que la linguistique ne fait habituellement pas partie du programme scolaire du secondaire et ceci, ni dans les cours de langues, ni dans des cours spécifiques, c'est seulement à l'université que les étudiants ont une première approche de cette discipline.

Alors que les études littéraires leur sont familières à partir des petites classes, la linguistique est ressentie comme une matière tout à fait nouvelle. Parmi toutes les nouveautés que représente la vie académique, l'initiation à la linguistique constitue non pas un obstacle, mais malgré tout un seuil et ceci tout au début des études, puisque ces cours sont prévus pour la $1^{\text {re }}$ ou la $2^{\mathrm{e}}$ année d'études. Au caractère nouveau et inhabituel de la linguistique, s'ajoute l'impression d'un degré d'abstraction que les étudiants de philologie n'ont pas rencontré jusque-là et qu'il est nécessaire, mais pas toujours facile, de corriger ou d'atténuer.

Il en résulte la nécessité d'utiliser tous les moyens didactiques adéquats pour transmettre le message d'une science «faisable », applicable et utile, y compris pour ce qui concerne les instruments des nouvelles technologies.

À côté d'exemples les plus concrets possible pouvant illustrer les phénomènes de la langue pour en faire « avaler » les régularités et structures, il s'agit surtout de faire acquérir aux étudiants l'aptitude à comprendre les descriptions de la langue (donc les 
règles linguistiques). L'obstacle prioritaire réside dans la terminologie linguistique dont la forme inhabituelle et le contenu souvent bien délimité et défini demandent évidemment un effort qui, pour une partie des étudiants, ne correspond pas à l'idée qu'ils se font d'études philologiques.

Les enseignants universitaires se voient donc dans l'obligation de s'adresser à la " didactique du tertiaire » pour réussir cette tâche difficile qui constitue en effet un défi intéressant.

L'un des moyens susceptibles de résoudre ce problème consiste à valoriser les apports de la technologie, informatique ou pédagogique, qui se trouvent en général à la disposition des enseignants... et des enseignés. Parmi ces apports figure le HTML.

\section{La technologie du HTML}

Quelles sont les implications techniques de la réalisation des projets linguistiques? Le langage HTML (Hyper Text Markup Language) est le langage de programmation des documents actuellement utilisé sur Internet. Il s'agit de documents-textes à qui l'on donne certaines caractéristiques à l'aide de Tags (balises). Ainsi, nous pouvons attribuer des caractéristiques complémentaires à certains extraits ou tout simplement à certains mots d'un texte.

Les programmes de traitement de texte couramment utilisés nous permettent déjà de mettre en valeur les informations importantes en les écrivant en italique ou en gras. HTML, dans sa version la plus simple, permet à l'utilisateur de relier entre elles des informations venant de différents documents. A l'aide de balises particulières, il définit des mots interactifs qui créent des liens avec d'autres documents. Cette faculté de relier plusieurs documents, même sans connexion à Internet, permet de créer un réseau multidimensionnel d'informations. Ce faisant, nous quittons totalement la vision linéaire que nous connaissons lorsque nous consultons des livres ou des revues. Nous entrons dans un réseau d'informations sans nous rendre compte de la complexité des enchaînements.

Les besoins techniques préalables sont peu nombreux. Pour programmer ces documents et les balises qui en font partie, il suffit en fait de disposer d'un simple éditeur de texte. Le seul problème est de connaître le langage HTML avec ses commandes et sa syntaxe. Même si les éditeurs de logiciels livrent des programmes de plus en plus simples (notamment avec la technologie WYSIWYG - What you see is what you get) et facilitent ainsi l'élaboration des documents HTML, il est important de préciser qu'HTML est un langage de programmation et, qu'à la base, il ne peut être lu que par des personnes ayant suivi un enseignement dans ce domaine.

Avant d'aller plus loin et pour ne favoriser aucun éditeur, il est utile de préciser que tous les logiciels de programmation de pages HTML sont utilisables, même si, pour des raisons pratiques évidentes, nous nous limiterons à l'utilisation du Composer qui fait partie du Netscape Communicator que l'on peut se procurer gratuitement. 


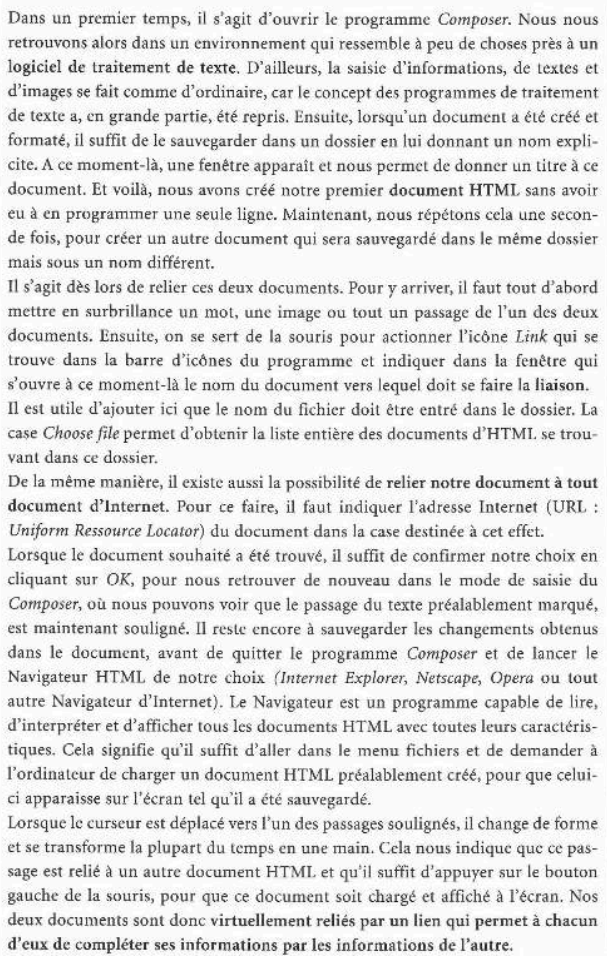

Pour examiner l'utilisation des

potentialités du langage HTML, qui repose sur la structuration des informations en petites unités isolées, prenons l'exemple de la définition d'un terme inconnu extraite d'un dictionnaire.

Il est facile de se représenter la succession des informations comme une chaîne d'informations. Le premier maillon de cette chaîne est l'explication même de notre terme. Or, cette explication est souvent compliquée, pourvue d'abréviations et de renvois à d'autres termes. Ces renvois forment le deuxième maillon de notre chaîne d'informations et ainsi de suite.

Pour rendre ce principe plus clair, nous allons en retracer les étapes en prenant comme exemple un terme linguistique. La première étape consiste à choisir un dictionnaire linguistique dans une bibliothèque. Ensuite, il s'agit de trouver le terme dans le dictionnaire, sachant que les mots y sont classés par ordre alphabétique. Ce dictionnaire ne nous donnera probablement qu'une brève définition du terme choisi et c'est exactement à cet endroit que débute l'utilisation de l'hypertexte.

Lorsque la définition du terme comprend des renvois à d'autres mots, il est possible que l'on doive répéter les différentes étapes une deuxième, voire une troisième fois. La présentation linéaire des informations trouvées dans les livres ou textes imprimés, ne permet pas au lecteur de se représenter les liens existant entre elles. Le lecteur se voit donc obligé de chercher son chemin avant d'obtenir toutes les informations désirées. Cela nécessite un surcroît de recherche et de concentration qui brise le processus d'apprentissage. C'est exactement cela que la structure d'information multidimensionnelle permet d'éviter.

Le lecteur peut s'approprier les informations par étapes successives et à des niveaux de difficultés différents. Grâce à une division en petites unités et à une structure claire, le lecteur peut choisir de façon interactive les informations qui lui sont utiles et rejeter celles qui ne lui apportent rien de nouveau. 
Lorsque l'on crée ce genre de documents d'informations, il faut tenir compte du fait qu'il existe énormément de chemins différents pour arriver à un but et que c'est au lecteur de choisir son propre parcours. Il est donc important de faire en sorte qu'une chaîne d'informations ne se termine pas par une impasse.

L'objectif final est un réseau d'informations multidimensionnel dans lequel l'utilisateur peut se déplacer librement et de façon virtuelle d'un maillon de la chaîne d'information à un autre et décider ainsi lui-même du contenu de son apprentissage. L'enchaînement des documents divisés en paquets permet de fixer la direction dans laquelle l'utilisateur doit se déplacer durant son apprentissage. Mais à partir du moment où il existe suffisamment de liens dans un document, le lecteur peut décider librement de son itinéraire. S'il arrive qu'un chemin mène dans une fausse direction, il est important que l'utilisateur puisse s'en rendre compte le plus rapidement possible, afin de ne pas entrer inutilement en contact avec des informations n'ayant rien à voir avec le sujet.

Ayant déterminé un thème, il faut planifier les possibilités d'enchaînement des informations avant de créer les divers documents qui le composent. Il est donc important de se fixer comme objectif une structure de l'information qui puisse la rendre aussi interactive que séduisante. En effet, l'organisation linéaire de l'information rend l'apprentissage fastidieux et peu efficace ; il faut donc chercher à la découper en petites entités simples à assimiler et à les relier par un maximum de liaisons interactives. Une telle structure permet un apprentissage plus efficace et ciblé au maximum sur les besoins du lecteur.

Voilà, nous avons clairement défini les avantages de cette forme d'apprentissage et à présent il ne nous reste plus qu'à faire le premier pas vers l'hypertexte pour permettre au lecteur de faire des découvertes en se divertissant et de réaliser un voyage à travers le réseau d'informations. 


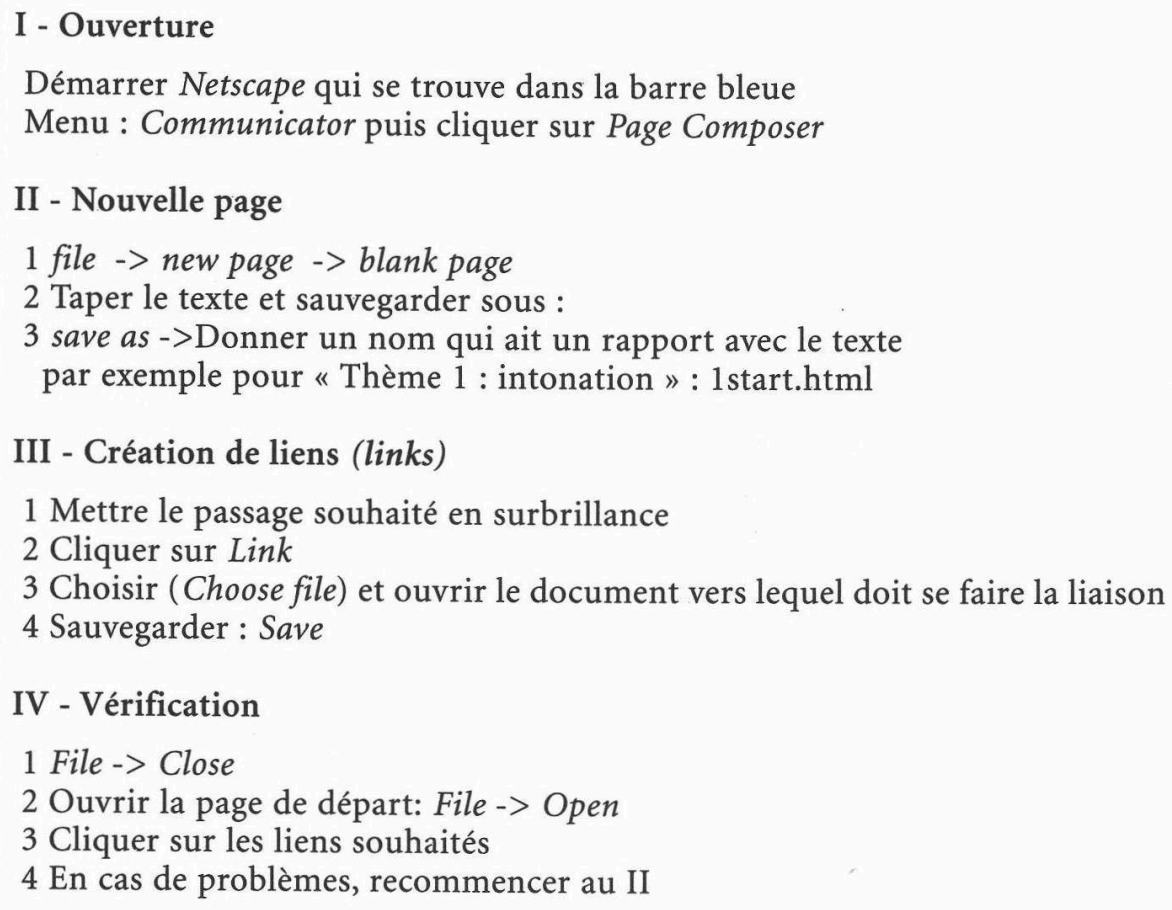

\section{Application du HTML}

L'un des moyens de promouvoir l'initiation à la linguistique est de zoomer sur la terminologie, dans l'objectif non de l'apprendre ou de la faire apprendre, mais de l'appliquer.

Un premier pas consiste à sélectionner les notions essentielles susceptibles de décrire les phénomènes qui caractérisent la communication, objet prioritaire de l'analyse. Les étudiants se voient donc confrontés aux différents modèles de la communication, impliquant des éléments tels que émetteur, récepteur, code, canal, message ; ce qui amène aux notions de langue, de parole, de compétence et de performance; il faut $\mathrm{y}$ ajouter les termes de situation de communication, de contexte, par opposition à cotexte. La connaissance du monde, en plus de la connaissance des contenus des signifiés des « mots ", a comme base la compréhension des structures sémantiques non seulement des mots ou signes, mais aussi des syntagmes, des phrases et finalement des textes. Cette connaissance du monde décide entre autres de l'interprétation des textes, écrits ou oraux ; la notion de " texte ", couvrant donc des modalités différentes, y compris le para-verbal et l'extra verbal (à l'oral et à l'écrit), est concurrencée par le terme de « discours ». Celui-ci désigne une communication dont les éléments, les énoncés, se combinent en une texture cohésive et cohérente d'actes de parole qui réussissent et, éventuellement, s'accomplissent. La structuration de ces discours est garantie par des procédures d'articulation, soit d'ouverture, soit de fermeture, soit de charnières textuelles, complétées par des procédés de prise ou d'abandon de parole par les communicateurs. Ainsi, la morphologie et la syntaxe, de même que ces deux disciplines, la phonétique/phonologie et la discipline graphique, s'intègrent dans la 
description globale de l'acte communicatif suivi qui, finalement, sera conçu comme une architecture, comme un ensemble à trois dimensions.

Ce tableau très succins des bases d'une initiation à la linguistique témoigne en effet d'un degré d'abstraction susceptible de provoquer les questions et les résistances esquissées plus haut. L'essentiel serait de mettre en contact ces catégories avec la vie réelle, c'est-à-dire de les faire comprendre en les appliquant à la description d'événements communicatifs. Mais pour y réussir, il faut montrer aux étudiants les rapports qui existent parmi ces termes et notions.

La complexité pluridimensionnelle de cet ensemble n'est pas facile à reproduire et donc à enseigner et dépasse souvent le cadre des cours dont on dispose en «Initiation à la linguistique », donc l'engagement, la compétence et l'horaire des étudiants. D'après nos expériences, le problème réside moins dans le nombre de termes (relativement modeste, en fin de compte) que dans la complexité des relations existant entre eux.

Le problème principal est de montrer la cohérence de cette terminologie et la complémentarité des notions. Ceci n'est pas impossible pour un enseignant qui utilise les moyens didactiques traditionnels comme le tableau noir ou le rétroprojecteur, mais cette tâche est radicalement facilitée par la technologie HTLM décrite plus haut, et ceci pour deux raisons :

- cette technique permet aux apprenants et aux enseignants de "voir» et de "manipuler » les rapports sémantiques entre les notions, en les concrétisant à l'aide de links (renvois);

- en outre, cette technique favorise le travail autonome des étudiants, ce qui leur permet de développer un comportement fort différent en comparaison avec l'attitude plutôt passive affichée face à un ensemble complexe et intransigeant de données abstraites.

\section{Quelques liens du réseau terminologique}

L'« arbre »: dans le cas de l'hypéronymie/l'hyponymie, il s'agit de termes qui s'organisent par « niveaux ». Ainsi, d'après la définition de Saussure, « langue » et « parole » sont les hyponymes de « langage » et forment donc deux branches partant d'un nœud commun, « langage ». On peut y ajouter « terme large », « terme étroit ». Les links peuvent aller soit vers l'hyperonyme, soit vers le co-hyponyme. Un autre exemple nous est fourni par la pragmalinguistique, dont la théorie centrale est la théorie des actes de langage ; un acte de langage ou acte langagier est un hypéronyme dont les hyponymes sont l'acte locutoire, l'acte illo-cutoire et l'acte perlocutoire, qui, de leur côté, constituent des co-hyponymes. Pour citer un dernier exemple : " modalité » est l'hypéronyme, «l'écrit », « l'oral » en sont les hyponymes. «Langue » n'est nullement identique à « compétence ", ni " parole » à « performance ». Ce ne sont donc pas des synonymes et pourtant un renvoi peut être utile, ne serait-ce que pour exprimer que ce sont des notions en quelque sorte proches, sans être synonymes. Voici un autre exemple, non moins compliqué pour la simple raison qu'il n'y a pas de synonymes dans le sens de «signes dont la signification est identique »; ainsi " texte ", qu'on utilise aujourd'hui dans le sens d'" ensemble cohérent de signes » dans la modalité de l'écrit aussi bien que de l'oral et en y insérant même le para verbal et l'extra verbal, est souvent compris comme objet d'une linguistique textuelle, tandis que « discours » désigne également un ensemble cohérent de signes mais en tenant plutôt compte de l'enchaînement d'actes langagiers. 
"Cohésion » et « cohérence » se trouvent dans une relation un peu plus complexe parce que ces termes se définissent différemment, selon les théories. «Cohérence » est souvent considéré comme le terme le plus vaste et englobe " cohésion » (qui fait appel aux seules marques morphosyntaxiques, tandis que « cohérence » comporte l'ensemble des marques linguistiques). "Cohésion » serait alors hyponyme de « cohérence ", sans avoir de co-hyponyme désignant les phénomènes complémentaires, où le nœud reste donc sans étiquette. Une autre interprétation consiste à dire que ce sont des cohyponymes, avec la spécificité qu'il n'existe pas d'hypéronyme lexicalisé. Ce serait la même relation que celle existant entre " cotexte » (environnement langagier) et " contexte » (environnement situationnel). «Cohésion » ainsi que « cohérence », par le fait qu'ils s'interprètent de façon différente, sont donc chacun des homonymes. Dans le cas du modèle de communication, les termes usuels énumérés plus haut désignent des parties d'un ensemble, à savoir de l'ensemble des éléments qui constituent ce modèle. "Canal », « émetteur ", " récepteur ", " code ", " message », etc. désignent ces parties et ne constituent ni des co-hyponymes ni des synonymes. Un cas parallèle : « signifiant » et « signifié », désignant chacun une partie de l'ensemble « signe ».

Il y a des termes qui, sans avoir de liens sémantiques, présentent néanmoins des identités ou des analogies de forme, ce qui est le cas par exemple pour « intention » et « intension », le dernier étant un terme sémantique, le premier désignant une notion pragmalinguistique. Ces deux termes, qui se ressemblent donc de par leur forme, prêtent à confusion, ce qui justifie sans aucun doute un link pour attirer l'attention sur ce phénomène.

Cette catégorisation, qui ne se veut pas exhaustive, illustre la complexité des rapports qui existent entre les termes de base et montre ainsi la nécessité de familiariser les étudiants avec ces problèmes.

La technologie HTLM permet d'appliquer la technique des renvois dans une mesure qui va bien au-delà de ce qui peut se réaliser avec les moyens traditionnels de présentation en classe. Cette technologie permet non seulement de présenter ces phénomènes linguistiques aux étudiants, mais leur donne qui plus est l'occasion de créer ces links eux-mêmes. Chacun d'eux s'occupe de l'un de ces termes et écrit sa page d'accueil en ajoutant aux termes traités des exemples, des remarques explicatives... et des links qui l'intègrent dans un réseau conceptuel. De cette façon, tous vont faire connaissance avec l'ensemble des termes, chacun découvre la totalité de la terminologie en partant d'un point de vue individuel et en utilisant les résultats de recherches des camarades. Cette approche aide à promouvoir la coopération parmi les étudiants; chacun d'eux a besoin du travail des autres et fournit de son côté des éléments dont se servent ses camarades. Cette réciprocité du travail, cette utilisation mutuelle permettent de juger de la valeur des résultats des recherches et soutient l'éveil d'un sens d'autoévaluation des travaux.

L'interdépendance du travail approfondit le sens de responsabilité de chacun et change un cours de linguistique en un groupe de travail où se constitue très rapidement un réseau de liens de coopération, analogue au réseau scientifique de termes linguistiques qui font l'objet de recherches et d'applications. 


\section{RÉSUMÉS}

Comment la «didactique du tertiaire » constitue-t-elle un moyen de surmonter les difficultés inhérentes à la discipline, pour de jeunes étudiants initiés à l'étude de la linguistique ? L'article fait état d'une démarche de travail en coopération et de mise en commun d'un réseau terminologique scientifique, propre à favoriser le travail en autonomie des étudiants et à concrétiser des notions abstraites.

\section{INDEX}

Index géographique : Allemagne

Mots-clés : nouvelles technologies, pédagogie, linguistique, langue allemande, TICE : technologies de l'information et de la communication en éducation

\section{AUTEURS}

\section{ALBERT RAASCH}

Professeur, institut d'études romanes, université de la Sarre, Sarrebruck, Allemagne

\section{BJÖRN KETTNER}

Étudiant en informatique, institut d'études romanes, université de la Sarre, Sarrebruck, Allemagne 